\title{
Multiplexed single-molecule flow-stretching bead assay for DNA enzymology
}

\author{
Ryanggeun Lee ${ }^{1, \#}$, Keunsang Yang ${ }^{2, \#} \mathcal{E}$ Jong-Bong Lee $e^{1,2, *}$ \\ ${ }^{1}$ Department of Physics, Pohang University of Science and Technology (POSTECH), Pohang 37673, ${ }^{2}$ School of Interdisciplinary Bioscience \\ and Bioengineering, POSTECH, Pohang 37673, Korea
}

\begin{abstract}
Single-molecule techniques have been used successfully to visualize real-time enzymatic activities, revealing transient complex properties and heterogeneity of various biological events. Especially, conventional force spectroscopy including optical tweezers and magnetic tweezers has been widely used to monitor change in DNA length by enzymes with high spatiotemporal resolutions of $\sim$ nanometers and $\sim$ milliseconds. However, DNA metabolism results from coordination of a number of components during the processes, requiring efficient monitoring of a complex of proteins catalyzing DNA substrates. In this min-review, we will introduce a simple and multiplexed single-molecule assay to detect DNA substrates catalyzed by enzymes with high-throughput data collection. We conclude with a perspective of possible directions that enhance capability of the assay to reveal complex biological events with higher resolution. [BMB Reports 2019; 52(10): 589-594]
\end{abstract}

\section{MULTIPLEXED SINGLE-MOLECULE FORCE SPECTROSCOPY}

Single-molecule studies have extensively explored a variety of biological problems by following individual molecules going through their biochemical or biophysical reactions (1-3). Single-molecule assays have provided real-time snap shots of molecular events, resolving molecular sub-populations, transient intermediate states, rare molecular events, and non-uniform kinetics in the enzymatic reaction (4). In contrast to direct observation of probe-labeled enzymes on DNA using fluorescence microscopy (5), the most commonly used force

*Corresponding author. Tel: +82-54-279-2095; Fax: +82-54-2793099; E-mail: jblee@postech.ac.kr

${ }^{\text {\#}}$ These authors contributed equally to this work.

https://doi.org/10.5483/BMBRep.2019.52.10.170

Received 9 July 2019

Keywords: Flow-stretching bead assay, Force spectroscopy, Multiplexed single-molecule assay, Single-molecule enzymology, Single-molecule technique spectroscopy, including optical tweezers (OT), magnetic tweezers (MT), and atomic force microscopy (AFM), can manipulate individual biomolecules by external forces in the range of $0.01-10,000 \mathrm{pN}$ to unravel structural and mechanical properties (6). The force spectroscopy has been also used to precisely measure displacement as a function of force with high spatiotemporal resolution of sub-nanometers and submilliseconds to detect enzyme activities on substrates in DNA replication, DNA repair, transcription, and translation (7-11).

However, traditional force spectroscopy is difficult to parallelize measurement at one time, limiting monitoring of enzymatic activity by multi-proteins due to inefficient in vitro reconstitution of the biological system of interest (12). To achieve high-throughput data collection, optical tweezers and magnetic tweezers have been modified to efficiently collect data in parallel. Although OT uses a focused laser beam to trap a polystyrene/silica bead linked to DNA, holographic light using a spatial light modulator can trap $\sim 100$ beads per field of view (13) (Fig. 1A). However, this multiplexed OT is complex and needs professional knowledge and high cost to build and operate. MT uses a magnetic force applied to a superparamagnetic bead linked to DNA molecules by a magnet to manipulate bead-DNA immobilized on the surface. To increase the number of DNA-tethered beads, an array of DNA molecules linked to beads is immobilized on the patterned surface of the imaging chamber ( 300 DNAtethered beads per field of view) (Fig. 1B) $(14,15)$. Recently, another high-throughput force spectroscopy, called acoustic force spectroscopy (AFS), was developed, which can detect tens-thousands of DNA molecules per field of view depending on the magnification of an objective lens (16). The AFS uses acoustic waves to trap polystyrene microspheres attached to surface tethered-DNA molecules, resulting in the extension of multiple DNA molecules immobilized on the surface in an upward direction to the surface (Fig. 1C). These multiplexed MT and AFS require complex instruments and a calibration profile of diffraction pattern images of beads for measurement of bead displacement due to the vertical motion of trapped beads to the imaging plane.

In this mini review, we describe a simple, robust, low cost, and multiplexed single-molecule force spectroscopy to study processive enzyme activities on stretched-DNA substrates 
A

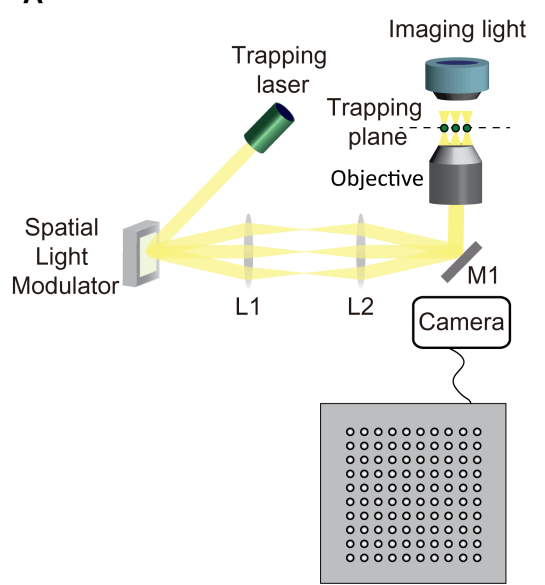

B

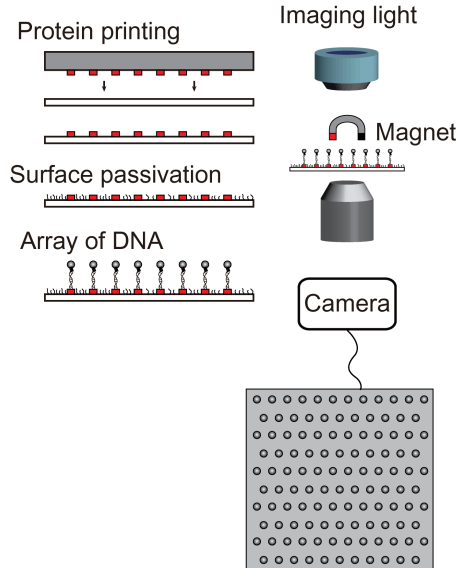

C

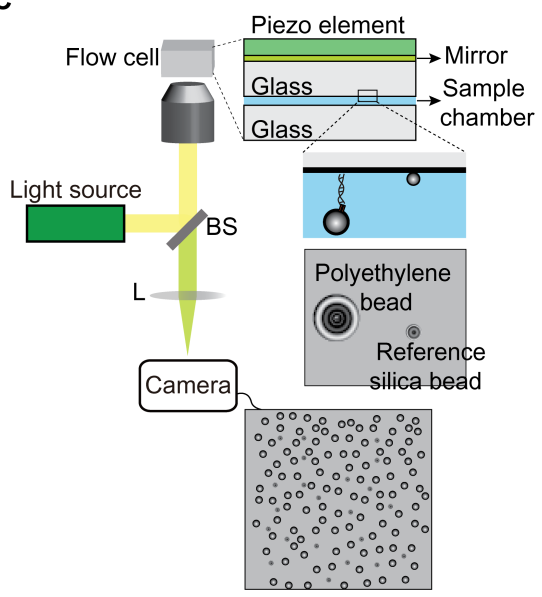

Fig. 1. Multiplexed single-molecule force spectroscopy. (A) Holographic optical tweezers. (B) Multiplexed magnetic tweezers. (C) Acoustic force spectroscopy.

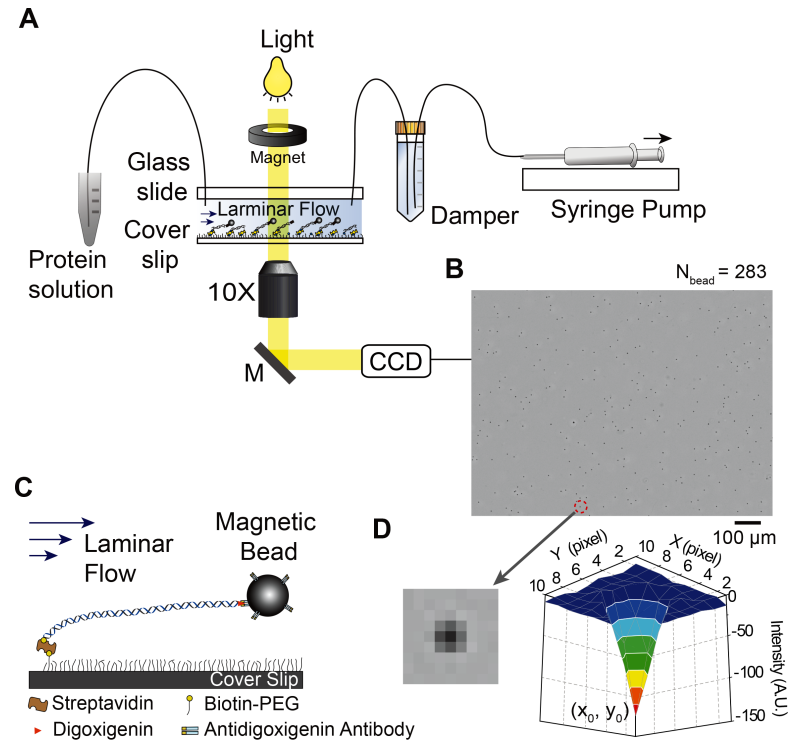

Fig. 2. Schematic representation of single-molecule flow-stretching bead assay. (A) A setup based on a conventional optical microscope. A buffer solution including target proteins flows through the flow chamber with constant rate by a syringe pump. (B) $\sim 300$ beads (2.8 $\mu \mathrm{m}$ in diameter) attached to tethered-DNA molecules on the surface under 10x magnification objective. (C) Bead-DNA is immobilized on the streptavidin-coated surface that is passivated with biotin-PEG/PEG (1:100). A laminar flow stretched bead-DNA. (D) Intensity profile of a bead image shows a Gaussian distribution.

using a hydrodynamic force, called flow-stretching bead assay.

\section{SINGLE-MOLECULE FLOW-STRETCHING BEAD ASSAY (smFS)}

\section{Overview of smFS}

To monitor individual DNA in the smFS, DNA molecules are immobilized on the surface passivated with biotin-PEG (polyethylene glycol) via biotin-avidin interactions while the opposite end with digoxigenin is attached to a superparamagnetic bead $(2.8 \mu \mathrm{m}$ in diameter) functionalized with anti-digoxigenin antibody. A steady buffer flow with a constant rate given by a syringe pump drags the bead linked to the tethered-DNA, resulting in extension of DNA (Fig. 2A). A 
damper made by a Falcon centrifuge tube that is filled with water and an air layer is installed between the syringe pump and flow chamber to filter high-frequency noises coming from mechanical fluctuation of the syringe pump (Fig. 2A). The position of beads is monitored to measure enzymatic activities on DNA substrates. Beads linked to immobilized-DNA molecules are visualized under a standard optical microscope and recorded by a charge coupled device (CCD) camera (Fig. 2A). Dark spots in the resulting image correspond to the beads (Fig. 2B). Optimal number of beads linked to DNA is $\sim 300$ per field of view under 10x objective. In the smFS, we can monitor enzyme activities on DNA substrates by measuring changes in the length of individual DNA molecules by imaging beads and tracking their position.

\section{Flow chamber construction}

A flow chamber was constructed with a biotin-PEG functionalized cover slip, PEG-coated glass slide, double-sided tape, and tubing (Fig. 2C). A pair of inlet/outlet holes is drilled on a glass slide before it is passivated with PEG. A $100 \mu \mathrm{m}$ thick double-sided tape is cut for a channel with $3 \mathrm{~mm}$ in width and $25 \mathrm{~mm}$ in length. Width and height of the channel must be maintained to perform experiments under an identical force once the external force is calibrated to flow rate by the syringe pump. A solution of streptavidin or neutravidin is evenly spread on the biotin-PEG cover slip and then washed with deionized water after 10 minutes of incubation. A chamber is made by putting the prepared cover slip and glass slide together with adhesive double-sided tape and then sealing with epoxy. Tubing is inserted into each inlet/outlet hole and then epoxy is applied to the junction between the hole and tubing. The flow chamber filled with a blocking buffer is ready to do a single-molecule flow-stretching experiment. For more detailed information on construction of the flow chamber, we refer to a demonstration movie published by the journal of visualized experiments (JoVE) (17).

\section{Localization of beads in a nanometer scale}

The position of a bead is determined by two dimensional (2D) Gaussian fit to the intensity profile of the bead (Fig. 2D). The 2D Gaussian function is defined as

$$
I(x, y)=I_{0}+A \exp \left[-\frac{\left(x-x_{0}\right)^{2}+\left(y-y_{0}\right)^{2}}{2 s^{2}}\right]
$$

Where $I_{0}$ is a constant due to background noises, $A$ is the amplitude of the fitted function, $s$ is the standard deviation of the fitted function, and $x_{0}$ and $y_{0}$ are the bead's fitted position. By taking all possible noise sources into account, the precision of $\left(x_{0}, y_{0}\right)$ is theoretically given by (18)

$$
\sigma=\sqrt{\frac{s^{2}}{N}+\frac{a^{2}}{12 N}+\frac{8 \pi s^{4} b^{2}}{a^{2} N^{2}}}
$$

where $N$ is the number of collected photons, $a$ is the pixel size of a CCD camera, and $b$ is the standard deviation of the background. The first term is photon shot noise, the second term is due to the finite pixel size of the CCD camera, and the last term is the background effect. This formula explicitly indicates that the more photons collected, the higher position

\section{A}

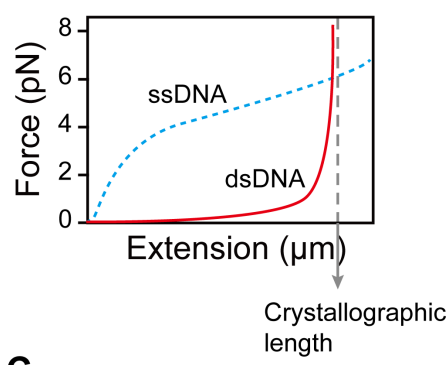

C

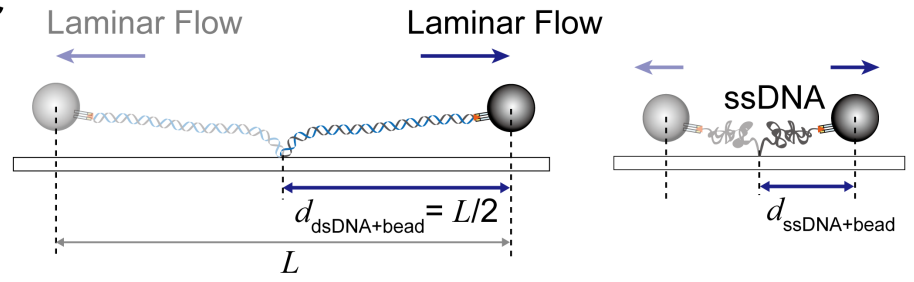

B

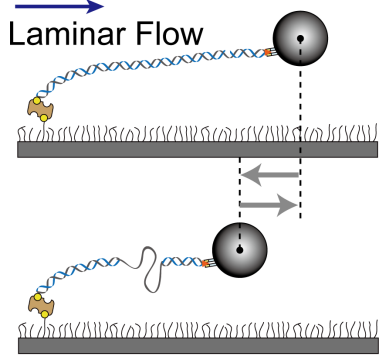

Fig. 3. Flow-stretching bead assay using different elastic properties between dsDNA and ssDNA. (A) Force-extension curve for dsDNA and ssDNA. (B) Transition of dsDNA (ssDNA) to ssDNA (dsDNA) is shortening (lengthening). (C) The scheme of the length measurement of dsDNA and ssDNA at a given force. 
A
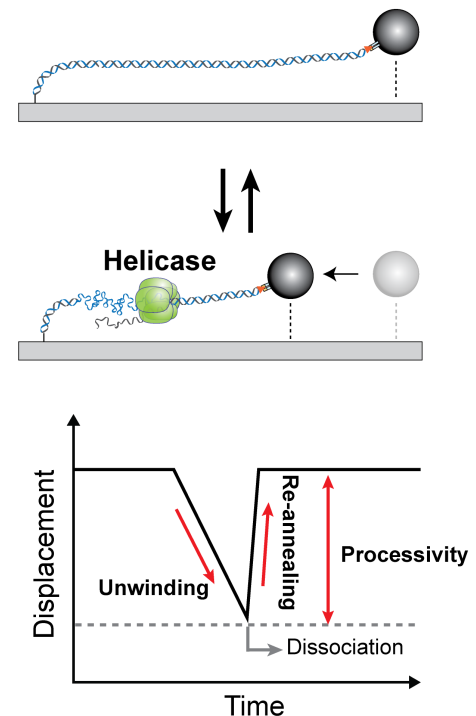

B

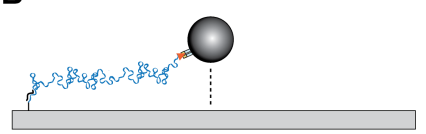

DNA
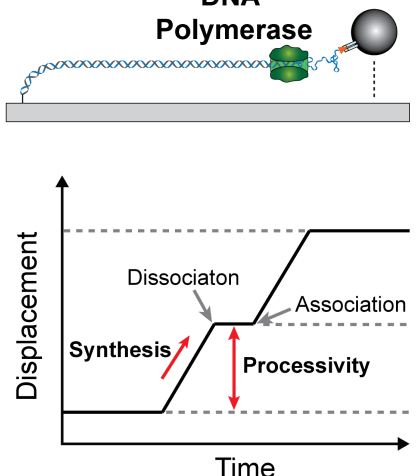

C
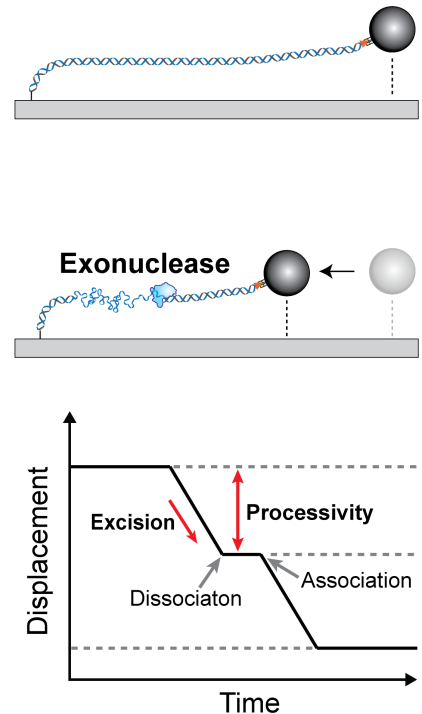

Fig. 4. Time trajectories of enzyme activities with flow-stretching bead assay. (A) Helicaese unwinds duplex DNA (dsDNA $\rightarrow$ ssDNA). Slope in time trajectory is the unwinding rate. Helicase dissociation drives reannealing of unwound strands (ssDNA $\rightarrow$ dsDNA). (B) The primer extension of DNA polymerases (ssDNA $\rightarrow$ dsDNA). (C) The strand excision by exonuclease (dsDNA $\rightarrow$ ssDNA).

accuracy is determined. A powerful tracking software is freely available at DiaTrack (www.diatrack.org).

\section{THE LENGTH CHANGE BETWEEN dsDNA AND ssDNA}

The smFS detects activities of DNA enzymes using the different elastic property of single-stranded (ssDNA) and double-stranded DNA (dsDNA). The ssDNA is more flexible (shorter persistence length) than dsDNA and easily forms secondary structure (hairpin-coil) by base pairing in the same strand. Thus, coiling of ssDNA results in shortening of dsDNA at low stretching forces $(<6 \mathrm{pN})$ (Fig. 3A). At forces higher than $6 \mathrm{pN}$ that is sufficiently strong to break the secondary structure, the ssDNA is extended longer than dsDNA because distance between base pairs in SSDNA is longer than that in the double-helical dsDNA (Fig. 3A). Therefore, conversion from dsDNA to ssDNA or vice versa can be monitored through the motion of beads in the opposite direction (a decrease in total DNA length) or in the same direction as the flow (an increase in total DNA length) (Fig. 3B).

Bead position on the nanometer scale obtained from the pixel size of the CCD camera (effective pixel size $=$ physical pixel size/magnification) is converted to the number of nucleotides (nt) transitioned from dsDNA (ssDNA) to ssDNA (dsDNA) by measuring the length of dsDNA and ssDNA. The ssDNA is prepared by denaturing the dsDNA. The dsDNA like 48.5 kilo-base pair $(\mathrm{kb})$ long $\lambda$-phage DNA is incubated in 2 $\mathrm{mM} \mathrm{NaOH}$ solution at $99^{\circ} \mathrm{C}$ for five minutes and then immediately quenched in a cold blocking buffer $\left(\sim 4^{\circ} \mathrm{C}\right)$ to prevent re-annealing. Distance $\left(d_{\mathrm{dsDNA}(\mathrm{ssDNA})}\right)$ between one end of dsDNA (ssDNA) (anchor point on the surface) and the center of a bead is determined by measuring half the distance $(L)$ between the centers of the bead at opposite directions of laminar flow (Fig. 3C). The length (I) of DNA is $I=d_{\mathrm{dsDNA}(\text { ssDNA) }}$ $-R_{\text {bead, }}$ where $R_{\text {bead }}$ is a bead radius. Conversion factor for transition between dsDNA and ssDNA is given by $\mathrm{nt} /\left(I_{\mathrm{dsDNA}}-\right.$ $I_{\text {sSDNA). }}$.

\section{APPLICATIONS IN DNA ENZYMOLOGY}

Since the single-molecule kinetics study of $\lambda$-exonuclease (19), the smFS has been successfully used for studying enzyme activities by multi-protein complexes in DNA replication (20-22) and DNA repair $(23,24)$. During DNA replication, helicase transforms from dsDNA to ssDNA by unwinding duplex DNA at the replication fork, resulting in shortening of DNA (Fig. 4A). Single-strand binding protein (SSB) is associated with resulting ssDNA before DNA synthesis by DNA polymerase and is also involved in DNA repair and recombination. DNA polymerase in the replisome synthesizes DNA on resulting ssDNA as a template, resulting in lengthening of DNA (Fig. 4B) $(20,25)$. Basically, time trajectory of the bead position can provide unwinding or synthesis rate and processivity of enzymatic activity (Fig. 4A, B). The strand excision by exonuclease is a crucial step to repair DNA, resulting in transition of dsDNA to ssDNA (Fig. 4C). Using the $\mathrm{smFS}$, Park et al. showed the proofreading activity of $E$. coli DNA polymerase III when it incorrectly synthesizes DNA (26). 
Jeon et al. revealed the mechanism of strand elimination by human Exol exonuclease through sequential coordination of components in DNA mismatch repair (24).

In addition to the detection of the transition of dsDNA (ssDNA) to ssDNA (dsDNA), since SSB binding to ssDNA extends ssDNA, but SSB-bound ssDNA is shorter than dsDNA, the smFS can visualize the SSB association and dissociation from $\operatorname{ssDNA}(21,23)$. A transient intermediate state, appearing as a pause in the time trajectory, was also detected; the primer synthesis by primase (20) and the stoppage of the Tus-Ter complex in the termination of DNA replication (27). The smFS can visualize in real time the conformational change in dsDNA; the dynamic loop formation and release of the lagging strand during the Okazaki fragment synthesis (21).

\section{PROSPECTS}

The smFS is a powerful toolbox to study enzymatic activities in a multi-protein complex in various DNA-associated biological events. Especially, it is suitable for highly processive enzymes. However, for higher resolution (more information) of molecular activity in the protein complex, the smFS can be combined with fluorescence imaging. The correlative system such as OT-fluorescence microscopy (28-36) and MT-fluorescence microscopy (37-40) can visualize directly fluorescently-labeled enzymes on the substrate as well as the product as change in DNA length. In fact, Loparo et al. developed the smFS combined with total internal reflection fluorescence (TIRF) microscopy to study the exchange of DNA polymerases in DNA replication (41). The micron-scale bead attached to DNA is not compatible with TIRF microscopy due to $100 \mu \mathrm{m}$ penetration depth of the evanescent wave. Instead, quantum dot (QD) to image change in DNA length was attached to a location close to the replication fork. Remaining DNA after the QD contributes to drag the QD, reducing thermal fluctuations of QD due to the small Stokes drag force on the QD. However, they need much faster flow rate (much more volume of proteins) and only observed less than 100 DNA-tethered QDs per field of view under 60x objective lens with high NA $(>1.2)$ for single fluorophore imaging. If the TIRF microscopy is replaced with highly inclined thin illumination (HiLo) with longer illumination depth, the larger bead can be used to increase spatial resolution. Immobilization of an array of DNA molecules on the patterned surface can dramatically increase the number of DNA substrates per field of view. We expect that the multiplexed correlative smFS with fluorescence imaging would greatly contribute to reveal unknown mechanisms in DNA-related biological events.

\section{ACKNOWLEDGEMENTS}

This study was supported by Global Research Lab Program through the NRF of Korea funded by the Ministry of Science and ICT (NRF-2017K1A1A2013241) (J.-B.L.).

\section{CONFLICTS OF INTEREST}

The authors have no conflicting interests.

\section{REFERENCES}

1. Miller H, Zhou Z, Shepherd J, Wollman AJM and Leake MC (2018) Single-molecule techniques in biophysics: a review of the progress in methods and applications. Rep Prog Phys 81, 024601

2. Zlatanova J and van Holde K (2006) Single-Molecule Biology: What Is It and How Does It Work? Mol Cell 24, 317-329

3. Deniz AA, Mukhopadhyay S and Lemke EA (2008) Single-molecule biophysics: at the interface of biology, physics and chemistry. J Royal Soc Interface 5, 15-45

4. Xie XS and Lu HP (1999) Single-molecule enzymology. J Biol Chem 274, 15967-15970

5. Juette MF, Terry DS, Wasserman MR et al (2014) The bright future of single-molecule fluorescence imaging. Curr Opin Chem Biol 20, 103-111

6. Neuman KC and Nagy A (2008) Single-molecule force spectroscopy: optical tweezers, magnetic tweezers and atomic force microscopy. Nat Methods 5, 491-505

7. Morin JA, Cao FJ, Lazaro JM et al (2012) Active DNA unwinding dynamics during processive DNA replication. Proc Natl Acad Sci U S A 109, 8115-8120

8. Abbondanzieri EA, Greenleaf WJ, Shaevitz JW, Landick R and Block SM (2005) Direct observation of base-pair stepping by RNA polymerase. Nature 438, 460-465

9. Wen JD, Lancaster L, Hodges C et al (2008) Following translation by single ribosomes one codon at a time. Nature 452, 598-603

10. Strick TR, Croquette V and Bensimon D (2000) Singlemolecule analysis of DNA uncoiling by a type II topoisomerase. Nature 404, 901-904

11. Fan J, Leroux-Coyau M, Savery NJ and Strick TR (2016) Reconstruction of bacterial transcription-coupled repair at single-molecule resolution. Nature 536, 234-237

12. Neuman KC, Lionnet $T$ and Allemand JF (2007) SingleMolecule Micromanipulation Techniques. Annu Rev Mater Res 37, 33-67

13. Padgett $M$ and Di Leonardo R (2011) Holographic optical tweezers and their relevance to lab on chip devices. Lab Chip 11, 1196

14. Ribeck $N$ and Saleh OA (2008) Multiplexed singlemolecule measurements with magnetic tweezers. Rev Sci Instrum 79, 094301

15. De Vlaminck I, Henighan T, van Loenhout MT, Burnham DR and Dekker C (2012) Magnetic forces and DNA mechanics in multiplexed magnetic tweezers. PLoS One 7, e41432

16. Sitters G, Kamsma D, Thalhammer G, Ritsch-Marte M, Peterman EJG and Wuite GJL (2015) Acoustic force spectroscopy. Nat Methods 12, 47-50

17. Kulczyk AW, Tanner NA, Loparo JJ, Richardson CC and van Oijen AM (2010) Direct observation of enzymes replicating DNA using a single-molecule DNA stretching assay. J Vis Exp 36, 1689 
18. Thompson RE, Larson DR and Webb WW (2002) Precise nanometer localization analysis for individual fluorescent probes. Biophys J 82, 2775-2783

19. van Oijen AM, Blainey PC, Crampton DJ, Richardson CC, Ellenberger T and Xie XS (2003) Single-molecule kinetics of lambda exonuclease reveal base dependence and dynamic disorder. Science 301, 1235-1238

20. Lee J-B, Hite RK, Hamdan SM, Sunney Xie X, Richardson CC and van Oijen AM (2006) DNA primase acts as a molecular brake in DNA replication. Nature 439, 621-624

21. Hamdan SM, Loparo JJ, Takahashi M, Richardson CC and van Oijen AM (2009) Dynamics of DNA replication loops reveal temporal control of lagging-strand synthesis. Nature 457, 336-339

22. Tanner NA, Hamdan SM, Jergic S et al (2008) Singlemolecule studies of fork dynamics in Escherichia coli DNA replication. Nat Struct Mol Biol 15, 998

23. Park J, Jeon Y, In D, Fishel R, Ban C and Lee JB (2010) Single-molecule analysis reveals the kinetics and physiological relevance of MutL-ssDNA binding. PLoS One 5, e15496

24. Jeon Y, Kim D, Martin-Lopez JV et al (2016) Dynamic control of strand excision during human DNA mismatch repair. Proc Natl Acad Sci U S A 113, 3281-3286

25. Jergic S, Horan NP, Elshenawy MM et al (2013) A direct proofreader-clamp interaction stabilizes the Pol III replicase in the polymerization mode. EMBO J 32, 1322-1333

26. Park J, Jergic S, Jeon $Y$ et al (2018) Dynamics of Proofreading by the E. coli Pol III Replicase. Cell Chem Biol 25, 57-66.e54

27. Elshenawy MM, Jergic S, Xu ZQ et al (2015) Replisome speed determines the efficiency of the Tus-Ter replication termination barrier. Nature 525, 394-398

28. Harada Y, Arai Y, Yasuda R et al (1999) Tying a molecular knot with optical tweezers. Nature 399, 446-448

29. Bianco PR, Brewer LR, Corzett M, et al (2001) Processive translocation and DNA unwinding by individual RecBCD enzyme molecules. Nature 409, 374-378

30. Sarangapani KK, Duro E, Deng Y et al (2014) Sister kinetochores are mechanically fused during meiosis $I$ in yeast. Science 346, 248-251

31. Chang M, Oh J, Kim Y, Hohng S and Lee JB (2017) Extended depth of field for single biomolecule optical imaging-force spectroscopy. Opt Express 25, 32189

32. Grashoff C, Hoffman BD, Brenner MD et al (2010) Measuring mechanical tension across vinculin reveals regulation of focal adhesion dynamics. Nature 466, 263-266

33. Hohng S, Zhou R, Nahas MK et al (2007) FluorescenceForce Spectroscopy Maps Two-Dimensional Reaction Landscape of the Holliday Junction. Science 318, 279-283

34. Zhou R, Kozlov Alexander G, Roy R et al (2011) SSB Functions as a Sliding Platform that Migrates on DNA via Reptation. Cell 146, 222-232

35. Comstock MJ, Whitley KD, Jia $\mathrm{H}$ et al (2015) Direct observation of structure-function relationship in a nucleic acid-processing enzyme. Science 348, 352-354

36. Heller I, Sitters G, Broekmans OD et al (2013) STED nanoscopy combined with optical tweezers reveals protein dynamics on densely covered DNA. Nat Methods $10,910-916$

37. Graham JS, Johnson RC and Marko JF (2011) Concentrationdependent exchange accelerates turnover of proteins bound to double-stranded DNA. Nucleic Acids Res 39, 2249-2259

38. Hugel T, Michaelis J, Hetherington CL et al (2007) Experimental Test of Connector Rotation during DNA Packaging into Bacteriophage $\varphi 29$ Capsids. PLoS Biol 5, e59

39. Madariaga-Marcos J, Hormeño $\mathrm{S}$, Pastrana $\mathrm{CL}$, Fisher GLM, Dillingham MS and Moreno-Herrero F (2018) Force determination in lateral magnetic tweezers combined with TIRF microscopy. Nanoscale 10, 4579-4590

40. Lee M, Kim SH and Hong SC (2010) Minute negative superhelicity is sufficient to induce the B-Z transition in the presence of low tension. Proc Natl Acad Sci U S A $107,4985-4990$

41. Loparo JJ, Kulczyk AW, Richardson CC and van Oijen A M. (2011) Simultaneous single-molecule measurements of phage $\mathrm{T7}$ replisome composition and function reveal the mechanism of polymerase exchange. Proc Natl Acad Sci U S A 108, 3584-3589 